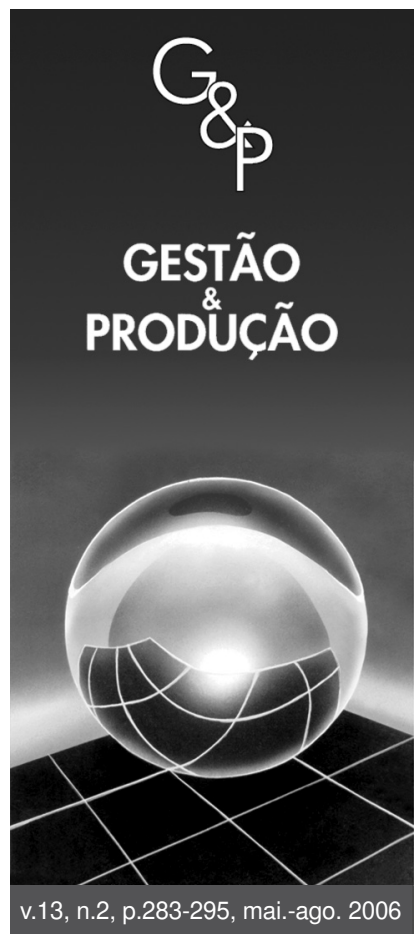

\title{
UM ESTUDO DA IMPLANTAÇÃO DE UM OTIMIZADOR DE MIX PARA O SETOR AGROPECUÁRIO
}

\author{
Guilherme Luís Roehe Vaccaro \\ Luís Henrique Rodrigues
}

Programa de Pós-Graduação em Engenharia de Produção e Sistemas - PPGEPS, Universidade do Vale do Rio dos Sinos - UNISINOS, Av. Unisinos, 950, Prédio 5, 3ํ Andar, Bairro Cristo Rei, CEP 93022-000, São Leopoldo, RS, e-mails: guilhermev@unisinos.br, Ihr@unisinos.br

Felipe Morais Menezes

Produttare Consultores Associados, Rua Engenheiro Afonso Cavalcanti, 54, Bairro Bela Vista,

CEP 90440-110, Porto Alegre, RS, e-mail: menezes@produttare.com.br

Recebido em 08/6/2005 Aceito em 17/4/2006

Resumo

O presente trabalho descreve uma abordagem de implantação de um otimizador de mix de produção em empresas do setor agropecuário, mais propriamente em frigoríficos. A implantação de um otimizador de mix envolve diversas áreas da organização, uma vez que esta é uma ferramenta sistêmica e que tem por objetivo gerar um plano mestre de produção orientado ao mercado, respeitando capacidades finitas agregadas e otimizando a lucratividade da empresa. $O$ artigo discute as dimensões envolvidas na implantação de um projeto dessa natureza, bem como os principais benefícios de sua implantação.

Palavras-chave: otimização de mix, planejamento de produção, processos divergentes.

\section{Introdução}

O Brasil ocupa uma posição estratégica no mercado frigorífico mundial. Em 2004, o complexo de carnes exportadas superou a marca de seis bilhões de dólares, sendo que cerca de cinco bilhões foram gerados pela exportação de carne de frango e bovina (ABIPECS, 2004). O País é o maior exportador, em volumes, de carne bovina e de frangos (ABIPECS, 2004), tendo superado as expectativas previstas para o ano de 2004 (ABEF, 2004). No entanto, por questões como cotas de exportação, ainda ocupa apenas a quarta posição como produtor de carne suína e contribui com apenas 3\% do volume exportado mundialmente (ABIPECS, 2004). As previsões são de que este quadro seja mantido no próximo biênio, com previsão de incremento anual médio de $5 \%$ nos volumes produzidos (ABEF, 2005).

Do ponto de vista comercial, a definição do mix de produção depende diretamente da compreensão das necessidades dos clientes, traduzida pelas previsões de demanda. A alta competitividade do mercado frigorífico e a pequena vantagem que o Brasil apresenta em relação a outros fornecedores em alguns setores do ramo frigorífico demandam vigilância no sentido do alinhamento adequado do mix de produção e vendas previsto em relação às expectativas e demandas dos mercados.

Do ponto de vista estratégico, a definição do mix de produção afeta a rentabilidade das empresas de duas formas: pela racionalização do uso de recursos, matériasprimas, transportes e outros elementos do ambiente produtivo; e pela indicação de qual combinação de produtos pode maximizar a receita líquida da empresa. Isso porque o mesmo frango (suíno, etc.), ao ser espostejado de formas diferentes, gera produtos finais diferentes, alterando a riqueza gerada a partir, essencialmente, da mesma matéria-prima.

Indústrias de produção conjunta e, mais particularmente, do setor frigorífico, apresentam como característica marcante a possibilidade de geração de uma grande quantidade de produtos finais a partir de um pequeno nú- 
mero de matérias-primas. Outra característica marcante desse tipo de indústria é que a geração de um conjunto de produtos implica na geração compulsória de outro conjunto de produtos. Essa característica de diversificação de produtos, ao longo da estrutura produtiva, torna estratégica e altamente impactante a questão do planejamento do mix de produção.

A adoção de um método e de ferramentas que permitam definir o mix de produção (e que, simultaneamente, levem em conta as características de produção conjunta do setor frigorífico, aspectos estratégicos como os mencionados anteriormente, restrições de capacidade produtiva, de suprimentos e de logística) faz-se necessária para agilizar a resposta das indústrias deste segmento frente a realinhamentos do mercado e outras incertezas críticas. $\mathrm{O}$ que diferencia a abordagem proposta de outras abordagens de definição de mix (Bayou e Reinstein, 2005; Corbett, 1998; Low, 1992; Lea e Fredendall, 2002) é a possibilidade de priorizar o adequado atendimento às necessidades de seus clientes, de identificar eventuais faltas de capacidade ou de matéria-prima em ambiente de planejamento e, em havendo excedentes, de maximizar o lucro das empresas pelo melhor aproveitamento da estrutura de produção divergente, característica desse tipo de indústria. A operacionalização desse método é o tema proposto neste artigo.

\section{Materiais e métodos}

Este artigo descreve um método para a definição do mix ótimo de produção e vendas, visando maximizar a lucratividade de indústrias de produção conjunta, tais como as do setor frigorífico. Por "ótimo" entende-se a melhor configuração de mix que atenda, simultaneamente, ao seguinte conjunto de critérios:

- Alinhamento às diretrizes da empresa, de atendimento ao mercado;

- Respeito a restrições da estrutura física da empresa, capacidades de recursos, características de qualidade dos produtos e disponibilidades de matérias-primas e insumos;

- Maximização da rentabilidade, expressa por elementos financeiros, tais como preço de venda diferenciado por item e mercado, despesas e custos variáveis, taxas de frete, etc.; e

- Minimização de estoques, considerando seu valor financeiro.

Chase e Aquilano (1995) apresentam a classificação VAT, que distingue as empresas com base na natureza do fluxo de materiais pela planta, considerando a estrutura dos roteiros de produção e a estrutura de materiais que compõem o produto. De uma forma geral, as empresas produtivas podem ser classificadas como $\mathrm{V}, \mathrm{A}$ ou $\mathrm{T}$, ou ainda como uma combinação desses três tipos, como por exemplo, I.

Empresas com processos produtivos do tipo V geralmente apresentam um pequeno número de matérias-primas, as quais são transformadas em uma gama variada de produtos finais. Estas são também chamadas de plantas de fluxo divergente, pela característica do fluxo dos produtos, como ilustrado na Figura 1. São exemplos desse tipo de empresas: empresas do ramo frigorífico, usinas de aço, refinarias de petróleo, empresas do ramo metalúrgico, indústrias de beneficiamento de madeira, etc. Dada a natureza de seu processo produtivo, esse tipo de empresa apresenta maior dificuldade na seleção do mix de produto, principalmente se considerada a dimensão estratégica de maximizar a rentabilidade do negócio da empresa.

Em empresas frigoríficas há ainda a dificuldade da definição da estrutura de produto, que pode ser representada por uma árvore composta por ramos compulsórios e por ramos alternativos. Os ramos compulsórios representam, por exemplo, cortes de componentes que são obrigatoriamente gerados. Exemplos de ramos compulsórios são pés, miúdos, cabeça, etc., para um suíno, ave ou bovino. Os ramos alternativos representam as decisões estratégicas dos programadores de produção, definindo quais cortes serão priorizados em uma dada produção. Exemplos de ramos alternativos são o envio de carcaças para congelamento (produto final) ou para espostejamento, gerando outros cortes, ou a opção de produção de frangos griller ou broiler. Nesse segmento, há também um outro tipo de decisão alternativa, que consiste na seleção de fórmulas para a fabricação de embutidos, empanados ou elaborados. Fórmulas diferentes podem dar ao produto final as mesmas características de paladar, consistência, etc., mas gerar impactos diferentes no aproveitamento dos cortes e dos insumos. Essas características tornam mais complexa a tarefa do gestor de produção, visto que

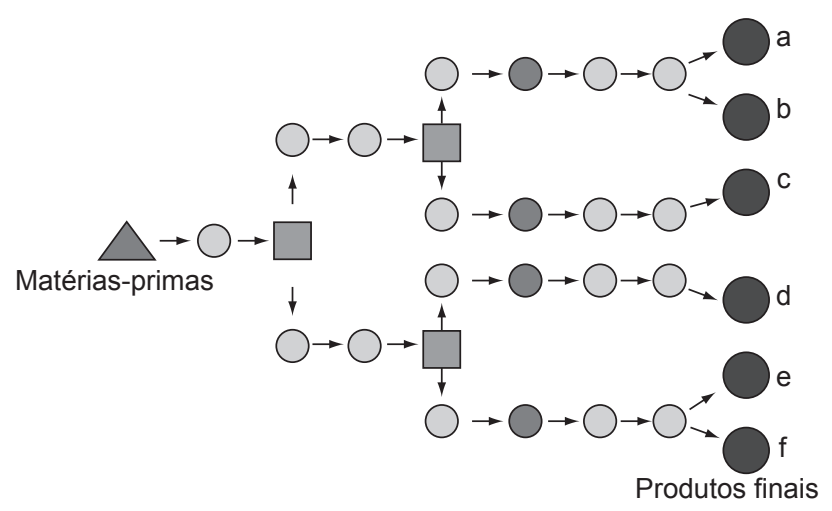

Figura 1. Esquema de uma planta tipo V. Fonte: Mansilha (2005), adaptado de Chase e Aquilano (1995). 
a integração de quais cortes realizar e quais fórmulas privilegiar impactam diretamente os níveis de inventário gerados.

O uso de ferramentas para o auxílio na definição de mix de produção é comum na pesquisa em Engenharia de Produção e é preconizado na atividade industrial (Bayou e Reinstein, 2005; Mansilha, 2005; Baldissera, 2003; Lea e Fredendall, 2002; Rodrigues et al., 2002; Antunes Jr. et al., 2001; Bercht, 1996; Chase e Aquilano, 1995; Goldratt, 1994; Rodrigues, 1994). O simples uso de uma ferramenta, porém, não é suficiente para garantir o sucesso de uma estratégia de definição otimizada de mix de produção. Bayou e Reinstein (2005) argumentam que a seleção do mix de produção é, em geral, uma decisão multinível, envolvendo critérios, pressupostos e objetivos conflitantes. O processo de implantação de uma ferramenta dessa natureza requer o envolvimento de pessoas em diferentes níveis da organização, estruturação de dados em informações adequadas para o uso da ferramenta e o uso de processos (Mansilha, 2005) para garantir a manutenção dos dados e a efetiva execução do plano de ação definido.

A presença de interações dinâmicas entre produtos, entre diferentes áreas da organização e entre decisões sucessivas ao longo do tempo (Bayou e Reinstein, 2005; Lea e Frendendall, 2002) também impacta no processo de seleção do mix de produção. Bayou e Reinstein (2005) sugerem o uso de um modelo hierárquico baseado em Lógica Difusa, enquanto Lea e Fredendall (2002) sugerem o uso de um modelo baseado em Programação Matemática para tratar dessas interações. A abordagem proposta neste artigo faz uso de um modelo de Programação Matemática, com função objetivo multicriterial, visando maximizar uma função de utilidade gerada a partir de elementos financeiros (maximização de rentabilidade, minimização de custos variáveis, etc.) e estratégicos (atendimento a clientes, uso de horas extras, etc.).

De modo a permitir uma visão ampla do escopo envolvido na abordagem proposta de seleção do mix ótimo de produção e vendas, os autores propõem a consideração de cinco dimensões:

1. Visão estratégica, orientada ao mercado;

2. Compreensão das características do setor de produção conjunta;

3. Incorporação dos conceitos preconizados pela Teoria das Restrições (Goldratt, 1994);

4. Incorporação das técnicas de otimização da Pesquisa Operacional; e

5. Incorporação dos conceitos de Gestão de Informação.

A seguir, esses elementos serão brevemente apresentados, de modo a compor o método proposto.

\subsection{Visão estratégica, orientada ao mercado}

Goldratt (1986) afirma que "a meta de uma empresa é ganhar dinheiro, hoje e no futuro". Um pensamento mais detido sobre essa máxima permite observar que "ganhar dinheiro, hoje e no futuro" preconiza a necessidade de manutenção de padrões de qualidade e excelência, de atendimento de necessidades específicas e alinhamento a demandas de clientes, e de racionalização das capacidades excedentes em função da maximização de lucros.

Classicamente, em indústrias de produção conjunta, a definição de mix de produção é "puxada" por estimativas mercadológicas. No entanto, essas estimativas são dadas, em geral, por meio de números fixos, baseados em séries históricas e na experiência das equipes de vendas. Esse modo de operação, apesar de efetivo, diminui a possibilidade de identificação de alternativas de maximização de rentabilidades. Em último caso, ao setor de Planejamento e Controle de Produção (PCP), resta definir se o conjunto de demandas apresentadas é viável ou inviável, dadas as capacidades de produção e de logística e as disponibilidades de suprimentos.

O método de implantação proposto sugere agregar a visão estratégica dos departamentos comerciais por meio de estimativas intervalares de demandas, sugerindo a definição de dois números para cada previsão em lugar de apenas um. O número menor, denominado "mínimo estratégico de vendas", indica a quantidade mínima que a empresa estima como relevante para a absorção de cada produto em cada mercado. O número maior, denominado "máximo de absorção de vendas", indica a quantidade máxima que poderá ser absorvida de cada produto em cada mercado a partir dos esforços de venda. Neste ponto, duas considerações são necessárias:

- O uso do mínimo estratégico de vendas e do máximo de absorção de vendas flexibiliza o processo de estimação e retira parte da criticidade do processo de estimação realizado pelas equipes de vendas, baseado em apenas um número por produto e mercado; e

- Se o mínimo estratégico de vendas e o máximo de absorção de vendas forem iguais, eles representarão o valor da estimativa de vendas classicamente utilizada; em outras palavras, para manter a coerência entre as visões propostas, estes valores deverão englobar a estimativa pontual classicamente utilizada.

A possibilidade de uso desses novos elementos é apenas uma parte do método proposto. No entanto é uma parte importante do processo, pois dá flexibilidade de decisão estratégica ao otimizador de produção sem ferir o alinhamento comercial estratégico da empresa. Durante a implantação, esses conceitos necessitam ser disseminados e bem compreendidos, uma vez que constituem uma das bússolas para a definição dos resultados a serem obtidos. De modo a tornar-se efetiva, essa bússola neces- 
sita ser completada pelos outros elementos apresentados e deve ser operacionalizada por meio de uma ferramenta computacional, conforme será discutido adiante.

\subsection{Compreensão das características do setor de produção conjunta}

$\mathrm{O}$ abate de um frango, por exemplo, origina, além das carnes, também pés, penas, sangue, vísceras, etc. No entanto, o destino dado a cada um desses elementos compulsórios no processo produtivo é estratégico de cada indústria, gerando alternativas de produtos semi-acabados, as quais poderão gerar novos ciclos de definição de itens compulsórios e alternativos até serem produzidos itens de venda efetiva ou componentes para formulados (embutidos, empanados, etc.). O conhecimento desse mecanismo de diversificação dos produtos semi-acabados faz parte da indústria de produção conjunta e do dia-a-dia dos programadores de produção de empresas desse setor. Situações similares ocorrem em empresas frigoríficas de suínos, de bovinos e em empresas do ramo petroquímico, por exemplo.

A incorporação do tratamento dessa diversidade se faz necessária para a obtenção de uma proposta de mix que tenha pretensões de otimização. Infelizmente, a capacidade humana de processar toda a diversidade de opções geradas nesse contexto divergente é insuficiente para propor um mix com essas características e em prazo hábil.

O método proposto define dois elementos que permitem modelar a divergência característica dos processos de abate e produção de cortes:

- Itens compulsórios: aqueles necessariamente gerados por um processo de abate ou corte; e

- Itens alternativos: aqueles que representam decisões estratégicas do setor de PCP, para o atendimento do mix com o melhor aproveitamento de matérias-primas e recursos.

A modelagem desses itens demanda o envolvimento de profissionais das áreas de PCP no sentido de construir estruturas formais de produto. É comum a existência de bancos de dados em empresas do setor frigorífico, assim como também é comum que o conhecimento efetivo de rendimentos de cortes e alternativas de produção seja domínio tão somente das pessoas, não havendo registro formal desses dados. Tipicamente, essa é uma informação que se encontra disseminada de maneira informal "na cultura da organização". Da mesma forma, é comum essa situação repetir-se com dados de roteiros e tempos de processo.

\subsection{Incorporação dos conceitos preconizados pela teoria das restrições}

A implantação de um sistema de planejamento da produção é freqüentemente observada como um "grande desafio", havendo a necessidade de se classificar e priorizar atividades. Nesse sentido, o método de implantação proposto sugere o uso de conhecimentos da Teoria das Restrições (TOC - Goldratt, 1994), tais como:

- Atuação sobre recursos restritivos de capacidade (Constrained Capacity Resources - CCR): aqueles que efetivamente restringem a capacidade de produção da empresa. Sob o prisma de que uma hora perdida em um CCR gera perdas reais para todo o sistema, a identificação de recursos CCR é fundamental para a definição da efetiva capacidade agregada das plantas produtivas. Além disso, o controle efetivo e a atuação sobre recursos CCR permitem reduzir as necessidades de levantamento de dados de tempos e de roteiros de produção; e

- Utilização da filosofia de custeio variável direto: O uso do conceito de "ganho" de um produto (preço de venda menos despesas totalmente variáveis diretas - Goldratt, 1994) permite identificar qual a efetiva contribuição de cada produto na maximização da rentabilidade da empresa. Custos variáveis indiretos são incorporados de forma indireta, a saber:

- Custos relativos à aquisição das matérias-primas e dos insumos são levados em conta, mas são incorporados a partir das necessidades de abate ou de compras identificadas na geração do mix ótimo de produção. Os custos unitários de aquisição de matérias-primas ou de insumos são utilizados no processo de otimização do mix; e

- Custos logísticos, de transporte entre unidades ou de estocagens, seguem as mesmas características dos custos de aquisição acima descritas.

O processo de custeio da Teoria das Restrições é bastante flexível e não se opõe - e não deve se opor - à existência de mecanismos tradicionais de custeio contábil, necessários para questões fiscais, por exemplo. Globalmente, a conta gerada pela "soma das margens, com despesas rateadas em cada produto" e a conta "total de receitas - total de despesas" gerarão o mesmo resultado. O método apenas propõe que o custeio variável seja usado para identificar os produtos mais atrativos do ponto de vista comercial e produtivo, sem depreciá-los pelo rateio da estrutura física, por exemplo, que deverá ser mantida, havendo ou não produção.

Para viabilizar a aplicação desses conceitos, o envolvimento dos profissionais de controladoria é fundamental, permitindo a definição da segunda bússola na busca de um mix de produção alinhado às tendências mercadológicas e que efetivamente resulte no maior lucro para a empresa como um todo.

\subsection{Incorporação das técnicas de otimização da pesquisa operacional}

As informações geradas nas diversas áreas da empresa envolvidas com o planejamento otimizado da produção devem ser validadas (no sentido de sua fidelidade e con- 
fiabilidade) e verificadas (no sentido de serem corretamente informadas à ferramenta de suporte). Isso porque elas formam a base para a geração de um modelo matemático de otimização que tem por objetivo maximizar a rentabilidade líquida da empresa, atendendo às previsões de demanda indicadas e às capacidades das plantas de fabricação. O modelo gerará um mix ótimo de produção vinculado aos dados que lhe foram informados, sugerindo decisões com base nesses dados. Para sugerir decisões úteis para a empresa, é necessário que o modelo receba dados exatos e realistas. Durante a implantação de uma solução dessa natureza o espírito de comprometimento com cada dado informado é fundamental, devendo este ser disseminado desde os operadores responsáveis por coletas de tempos até os mais altos níveis de gestão da empresa. Nesse sentido, o apoio da alta gerência da empresa é fundamental, uma vez que sua demonstração de comprometimento será refletida nos níveis operacionais.

\subsection{Incorporação dos conceitos de gestão de informação}

A abordagem metodológica se faz presente não apenas pelos referenciais teóricos, mas também a partir do provimento de meios que permitam sua utilização de forma sistemática. Sob esse aspecto, o método proposto necessita da existência de cinco características fundamentais da Gestão da Informação:

- Pessoas: as principais beneficiárias do método proposto. O provimento dos dados e a análise dos resultados propostos dependem da participação de funcionários. Em contrapartida, os benefícios gerados pela maior rentabilidade da empresa são sentidos por toda a empresa;

- Ferramentas de software e bancos de dados: necessários para incorporar a complexidade do sistema produtivo;

- Hardware: necessário para suportar as ferramentas de software e bancos de dados;

- Processos: fundamentais para a implantação e a manutenção do método, permitindo a visão da sequiência e dos efeitos de cada tarefa de um ponto de vista mais amplo; e

- Comunicação: transmissão de conceitos de Engenharia de Produção, de modelagem de recursos e de processos produtivos, entre outros, por meio de workshops, com foco em situações reais do setor.

\subsection{0 modelo de otimização}

Para dar suporte ao processo de seleção do mix ótimo de produção e vendas, um modelo multicriterial baseado em técnicas de Pesquisa Operacional, mais especificamente a Programação Matemática, foi desenvolvido. O modelo conceitual foi baseado no acompanhamento de casos em empresas do setor frigorífico (Rodrigues et al.,
2002), tendo sido realizadas entrevistas com diferentes integrantes de empresas e, após uma etapa de análise, identificadas as classes de restrições mais representativas a serem incorporadas no modelo de otimização:

- Alinhamento ao mercado;

- Estruturas do produto e necessidades físico-químicas de produtos formulados;

- Necessidades de fornecimento de matérias-primas; e

- Capacidades de produção nos CCRs.

Com base na identificação dessas classes de restrições, foi desenvolvido o modelo conceitual, considerando as seguintes variáveis de decisão:

$\mathrm{P}_{\mathrm{i}, \mathrm{m}}$ : quantidade em mil $\mathrm{kg}$ do produto i no período $\mathrm{m}$; $\mathrm{MP}_{\mathrm{i}, \mathrm{j}, \mathrm{m}}$ : quantidade em mil kg da matéria-prima j usada no produto i no período $\mathrm{m}$;

$\mathrm{B}_{\mathrm{i}, \mathrm{r}, \mathrm{m}}$ : número de bateladas do produto i no recurso $\mathrm{r}$ no período $\mathrm{m}$;

$\mathrm{V}_{\mathrm{i}, \mathrm{m}}$ : quantidade em mil $\mathrm{kg}$ do produto i vendida no período $\mathrm{m}$;

$\mathrm{E}_{\mathrm{i}, \mathrm{m}}$ : quantidade em mil $\mathrm{kg}$ do produto i estocada no período m;

$\mathrm{C}_{\mathrm{i}, \mathrm{m}}$ : quantidade em mil $\mathrm{kg}$ do produto i comprada no período $\mathrm{m}$; e

$\mathrm{F}_{\mathrm{i}, \mathrm{m}}$ : quantidade em mil $\mathrm{kg}$ de falta para o produto i no período $\mathrm{m}$.

Em termos de modelagem específica, as classes de restrições, são assim formuladas:

\subsubsection{Restrições de alinhamento ao mercado}

Cada produto final terá a sua quantidade vendida limitada ao máximo de mercado. O mínimo estratégico de vendas é representado pelos valores MIN $_{\mathrm{i}, \mathrm{m}}$ e o máximo de absorção de vendas, pelos valores $\mathrm{MAX}_{\mathrm{i}, \mathrm{m}}$

$$
\begin{aligned}
& V_{i, m} \leq \operatorname{MAX}_{i, m} \\
& V_{i, m} \geq \operatorname{MIN}_{i, m}+F_{i, m}
\end{aligned}
$$

\subsubsection{Restrições de estrutura de produto}

Para os itens formulavdos é necessário determinar a quantidade de cada componente

$$
\sum_{\mathrm{j}} \mathrm{MP}_{\mathrm{i}, \mathrm{j}, \mathrm{m}}-\mathrm{QPD}_{\mathrm{i}} * \mathrm{P}_{\mathrm{i}, \mathrm{m}}=0
$$

em que os valores QPDi representam as somas das quantidades padrão para produção de um $\mathrm{kg}$ do produto formulado i. Também é necessário determinar as características de composição do formulado, em função de quantidades mínimas e máximas dos componentes 


$$
\begin{aligned}
& \mathrm{MP}_{\mathrm{i}, \mathrm{j}, \mathrm{m}}-\mathrm{MINQ}_{\mathrm{i}, \mathrm{j}} * \mathrm{P}_{\mathrm{i}, \mathrm{m}} \geq 0 \\
& \mathrm{MP}_{\mathrm{i}, \mathrm{j}, \mathrm{m}}-\mathrm{MAXQ}_{\mathrm{i}, \mathrm{j}} * \mathrm{P}_{\mathrm{i}, \mathrm{m}} \leq 0
\end{aligned}
$$

em que os valores $\mathrm{MINQ}_{i, j}$ e $\mathrm{MAXQ}_{\mathrm{i}, \mathrm{j}}$ representam as quantidades mínima e máxima do componente que deve estar presente em um $\mathrm{kg}$ do produto i. Finalmente, são necessárias restrições para garantir a manutenção das características físico-químicas dos produtos

$$
\begin{aligned}
& \sum_{j} \operatorname{PDR}_{\mathrm{j}, \mathrm{k}} * \mathrm{MP}_{\mathrm{i}, \mathrm{j}, \mathrm{m}}-\mathrm{MINC}_{\mathrm{i}, \mathrm{k}} * \mathrm{P}_{\mathrm{i}, \mathrm{m}} \geq 0 \\
& \sum_{\mathrm{j}} \mathrm{PDR}_{\mathrm{j}, \mathrm{k}} * \mathrm{MP}_{\mathrm{i}, \mathrm{j}, \mathrm{m}}-\mathrm{MAXC}_{\mathrm{i}, \mathrm{k}} * \mathrm{P}_{\mathrm{i}, \mathrm{m}} \leq 0
\end{aligned}
$$

em que os valores $\mathrm{PDR}_{\mathrm{j}, \mathrm{k}}$ representam os padrões físicoquímicos da característica $\mathrm{k}$ no componente $\mathrm{j}$ e os valores $\mathrm{MINC}_{\mathrm{i}, \mathrm{k}}$ e $\mathrm{MAXC}_{\mathrm{i}, \mathrm{k}}$ representam as quantidades mínima e máxima da característica $\mathrm{k}$ no produto $\mathrm{i}$.

Para os itens de corte, os componentes devem ser tratados de acordo com as estruturas compulsórias ou alternativas. Itens de estrutura compulsória são derivados de cada item básico por meio de rendimentos específicos:

$$
\mathrm{RND}_{1 \mathrm{i}, \mathrm{i} 2} * \mathrm{P}_{\mathrm{i} 1, \mathrm{~m}}-\mathrm{P}_{\mathrm{i} 2, \mathrm{~m}}=0
$$

em que os valores $\mathrm{REN}_{\mathrm{i} 1 \text {, i2 }}$ representam o rendimento do corte de i1 para i2. Itens de estrutura alternativa são produzidos por um balanço de massas a partir do item básico:

$$
\mathrm{P}_{\mathrm{i} 1, \mathrm{~m}}-\sum_{\mathrm{i} 2} \mathrm{P}_{\mathrm{i} 2, \mathrm{~m}}=0
$$

Finalmente as quantidades produzidas necessitam ser transferidas entre os períodos, por um balanço de massas:

$$
\mathrm{P}_{\mathrm{i}, \mathrm{m}}+\mathrm{E}_{\mathrm{i}, \mathrm{m}}+\mathrm{V}_{\mathrm{i}, \mathrm{m}}-\mathrm{E}_{\mathrm{i}, \mathrm{m}-1}-\mathrm{C}_{\mathrm{i}, \mathrm{m}}=0
$$

\subsubsection{Restrições de necessidades de fornecimento de matérias-primas}

As necessidades de cada matéria-prima são as necessidades estabelecidas nas restrições anteriormente descritas, somadas adequadamente:

$$
\mathrm{P}_{\mathrm{j}, \mathrm{m}}-\sum_{\mathrm{i}} \mathrm{MP}_{\mathrm{i}, \mathrm{j}, \mathrm{m}}=0
$$

\subsubsection{Restrições de capacidades de produção nos CCRs}

Para definir a necessidade de um recurso que trabalha com bateladas é necessário que se calcule o número de bateladas necessárias para que toda a produção do mês seja contemplada:

$$
\mathrm{QPR}_{\mathrm{i}, \mathrm{r}, \mathrm{m}} * \mathrm{~B}_{\mathrm{i}, \mathrm{r}, \mathrm{m}}-\mathrm{P}_{\mathrm{i}, \mathrm{m}}>0
$$

em que os valores $\mathrm{QPR}_{\mathrm{i}, \mathrm{j}, \mathrm{m}}$ representam a quantidade do produto i processada por batelada do recurso $r$ no período $\mathrm{m}$. Uma vez definida a quantidade de bateladas a se- rem realizadas, restringe-se a necessidade total do recurso à sua capacidade:

$$
\sum \mathrm{TOP}_{\mathrm{i}, \mathrm{r}} * \mathrm{~B}_{\mathrm{i}, \mathrm{r}, \mathrm{m}}-\mathrm{RHX}_{\mathrm{r}, \mathrm{m}}-\mathrm{C}_{\mathrm{r}, \mathrm{m}} \leq 0
$$

em que os valores $\mathrm{TOP}_{\mathrm{i}, \mathrm{r}}$ representam os tempos padrão de operação de uma batelada do produto i no recurso $r$, os valores $\mathrm{C}_{\mathrm{r}, \mathrm{m}}$ e $\mathrm{RHX}_{\mathrm{r}, \mathrm{m}}$ representam os valores de capacidade normal e de horas extras disponíveis para o recurso $\mathrm{r}$ no período $\mathrm{m}$.

Para recursos que trabalham com valores unitários, a restrição de capacidade é simplesmente

$$
\sum_{\mathrm{i}} \mathrm{TOP}_{\mathrm{i}, \mathrm{r}} * \mathrm{P}_{\mathrm{i}, \mathrm{m}}-\mathrm{RHX}_{\mathrm{r}, \mathrm{m}}-\mathrm{C}_{\mathrm{r}, \mathrm{m}} \leq 0
$$

Outros tipos de recursos podem ser agregados, assim como roteiros alternativos, sendo este tipo de restrição bastante flexível para adaptação de outras realidades do processo produtivo.

\subsubsection{Função objetivo}

A função objetivo representa a utilidade da decisão multicriterial, por meio de um conjunto de variáveis e de pesos conflitantes. Essencialmente, cada item vendido aumentará o lucro da empresa proporcionalmente a sua margem de contribuição. O uso de horas extras, estoques ou o não atendimento a mercados é devidamente penalizado por custos unitários de utilização ou de custos estratégicos estabelecidos de acordo com o caso analisado, bem como a necessidade de compra de matérias-primas de terceiros.

Este modelo conceitual foi implementado na forma de um aplicativo computacional de suporte à decisão denominado ProfMix - Profit Mix, da Produttare Consultores Associados (Rodrigues et al., 2002). Posteriormente, modelo e aplicativo foram acrescidos de outras funcionalidades tais como consideração de outros tipos de recursos, tipos diferenciados de estocagem, tipos diferenciados de matérias-primas, entre outros.

\section{Discussão e resultados}

O método de implantação proposto foi desenvolvido a partir de projetos acadêmicos (Rodrigues, 1994; Baldissera, 2003; Oenning, 2004; Mansilha, 2005) e validado comercialmente em empresas do setor frigorífico. $\mathrm{O}$ tempo de implementação varia com a complexidade da empresa (número de itens de venda, número de unidades produtivas, número de mercados atendidos, etc.) e com a disponibilidade de dados em sistemas corporativos. $\mathrm{O}$ acompanhamento de casos de implantação permite observar o seguinte perfil nas empresas do setor frigorífico:

- Independentemente do tamanho da empresa, há um monitoramento sistemático dos dados comerciais e de controladoria, com conhecimento refinado de informações dessa natureza. Parte das empresas faz uso de sistemas 
de monitoramento baseados em planilhas eletrônicas. Grande parte das empresas faz uso de sistemas integrados de gestão com bases de dados corporativas;

- Dados de produção são gerenciados em bases locais ou por conhecimento tácito. É comum a ausência de informações sistematizadas, em bancos de dados, sobre as decisões compulsórias e alternativas tomadas sobre as estruturas de corte, principalmente no que se refere a itens semi-acabados. A gestão da produtividade dos recursos críticos é, em geral, controlada de forma sistemática, mas não é rara a ausência de informações sistematizadas sobre roteiros de produção; e

- Para o caso da indústria de aves, os dados de fomento são geralmente mantidos com alto refinamento, tendo em vista a suscetibilidade desse tipo de matéria-prima a questões tais como o tempo de permanência em criação e a linhagem genética. Para as demais indústrias do setor, este é um conhecimento em formação.

A implementação do método é desenvolvida a partir da definição de processos de implantação, como os apresentados nas Figuras 2 e 3, baseados na metodologia de mapeamento de processos (Davenport, 1994). Nessas figuras, eventos são representados por hexágonos, ações por retângulos de cantos arredondados, materiais ou informações por retângulos de cantos retos e pessoas responsáveis pelos processos por elipses. Outros processos são representados por retângulos decorados por hexágonos. Há ainda conectores lógicos, "e" $(\wedge)$, "ou" $(\vee)$ e "ou exclusivo" (xor). Um "e" indica que todas as ações geradas ou que todas as ações que o alimentam deverão ser cumpridas para que o processo continue fluindo. Um "ou" indica que pelo menos uma das ações geradas deverá ser concluída para que o processo continue fluindo e um "ou exclusivo" indica que somente uma das ações geradas deverá ser cumprida. O mapeamento de processos de implantação permite disseminar a visão contextual de cada ação entre os colaboradores do projeto, facilitando sua participação, e auxilia na redução da variabilidade dos tempos de execução de tarefas. O objetivo é dar uma visão mais ampla das atividades do projeto de implantação, permitindo que os colaboradores ajustem os tempos de execução sem grandes impactos nos prazos globais.

O primeiro processo a ser executado é o de Definição das equipes (Figura 2), pelo qual são nominados os colaboradores e definidos os gestores de cada processo de implantação, a saber: Coletar dados estruturais, Coletar dados comerciais, Coletar dados de controladoria, Coletar dados logísticos, Coletar dados de suprimentos e Ajustar sistemas corporativos. Para cada equipe, um gestor e uma lista de contatos deverá ser gerada. Uma lista consolidada de contatos deverá ser mantida pelo gestor principal do projeto.
Na coleta de dados estruturais prevê-se a modelagem das unidades produtivas, das unidades de estocagem, dos mercados, dos itens de produção (cortes e formulações), insumos e matérias-primas. Esse processo dá suporte para a coleta de dados de produção, tais como a definição das estruturas de produto (Figura 3 ) e a coleta de dados de recursos e de roteiros de fabricação. Esses processos carregam a responsabilidade de produzirem dados que representem o ambiente interno do PCP, de modo a modelar as estruturas de corte e desossa e de fabricação de embutidos, empanados, massadas, etc. Por meio dos trabalhos realizados observou-se que no setor frigorífico não é comum a existência de cadastros formais de itens intermediários de produção. A geração desses dados é importante do ponto de vista do melhor aproveitamento das matérias-primas em função da orientação do mercado, pois os processos de decisão tomados pelos analistas de PCP sobre esses itens devem ser também modelados para que o mix produzido seja efetivamente ótimo. Da mesma forma, as capacidades agregadas de produção, por meio de seus gargalos produtivos, devem ser corretamente informadas para que o método defina um modelo aderente à realidade das plantas de produção.

Os processos de Coleta de dados comerciais e Coleta de dados de controladoria são encarregados de identificar os elementos estratégicos de orientação ao mercado. Nesses processos os valores de mínimos estratégicos de vendas, de máximos de absorção de mercados, de composição de ganhos e de certificação de atendimento de mercados são definidos de modo a orientar a decisão do modelo de otimização. A qualidade dos dados gerados por esses processos é crucial no sentido do alinhamento estratégico do método aqui proposto, pois direciona as decisões. As experiências mostraram que o uso de valores de margens de contribuição não se mostrou tão efetivo quanto o uso de valores de ganhos, do ponto de vista da Teoria das Restrições. Por este motivo, a compreensão do impacto dos números fornecidos por parte dos colaboradores deve ser reforçada e constantemente reiterada pelos gestores do projeto. $\mathrm{O}$ mesmo se aplica aos demais dados coletados, sob outros pontos de vista.

Nos processos de Coleta de dados logísticos e de Coleta de dados de suprimentos os conjuntos de dados necessários são completados, sendo informados dados de custeio e capacidade de estocagem e de transporte, bem como disponibilidades de abates, de compras de matérias-primas e de insumos. Novamente, a abordagem de implementação por processos, aqui apresentada, permite que os profissionais de áreas não centrais em um projeto dessa natureza possam ser mais facilmente agregados como efetivos colaboradores.

Uma vez coletados os dados e formatados de maneira adequada, podem ser incorporados à base de dados da 


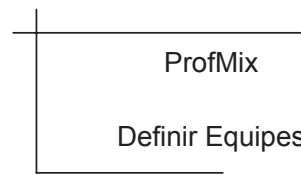

Um gesto por equipe, responsável por gerenciar as atividades, prazos e reportar relatórios aos gestores do projeto.

É conveniente a presença de um membro do corpo de TI em casa equipe, como facilitador de processos futuros.

\begin{tabular}{|l|}
\hline A equipe de dados \\
estruturais deve ser \\
formada por um \\
integrante de cada uma \\
das outras equipes.
\end{tabular}
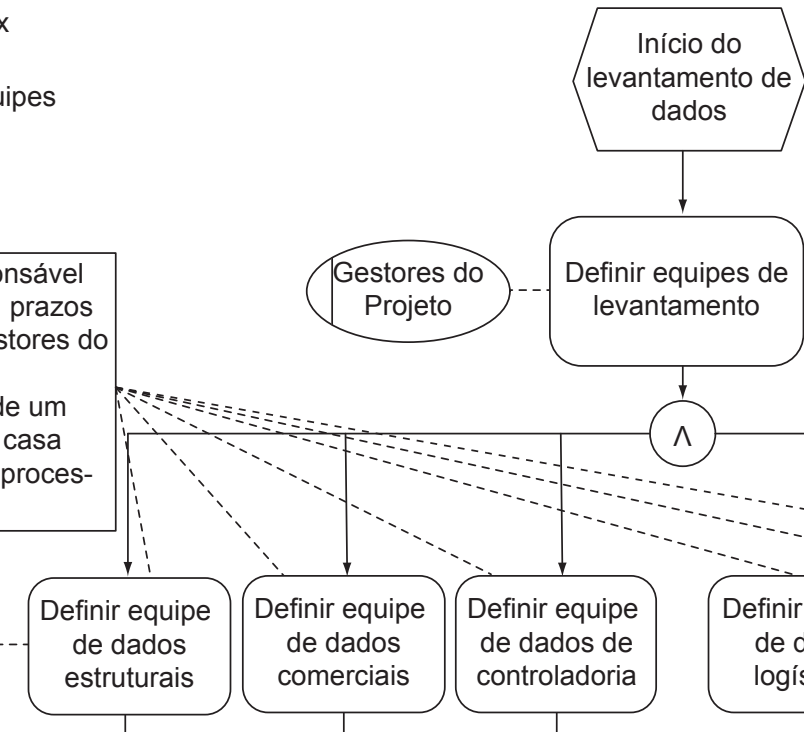

vel
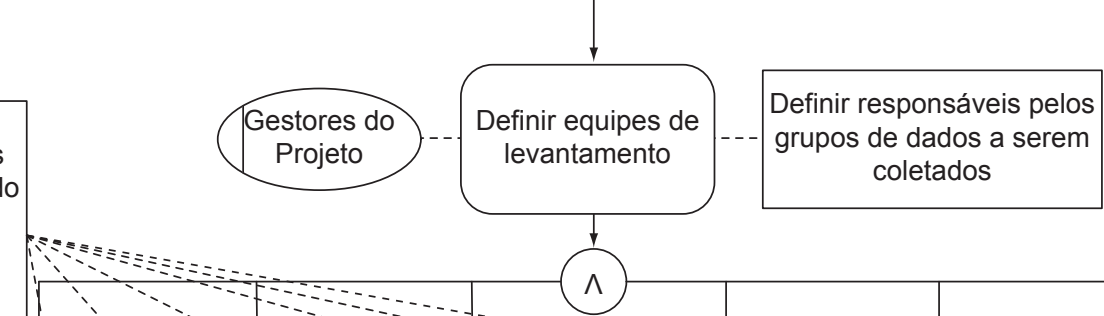
coletados

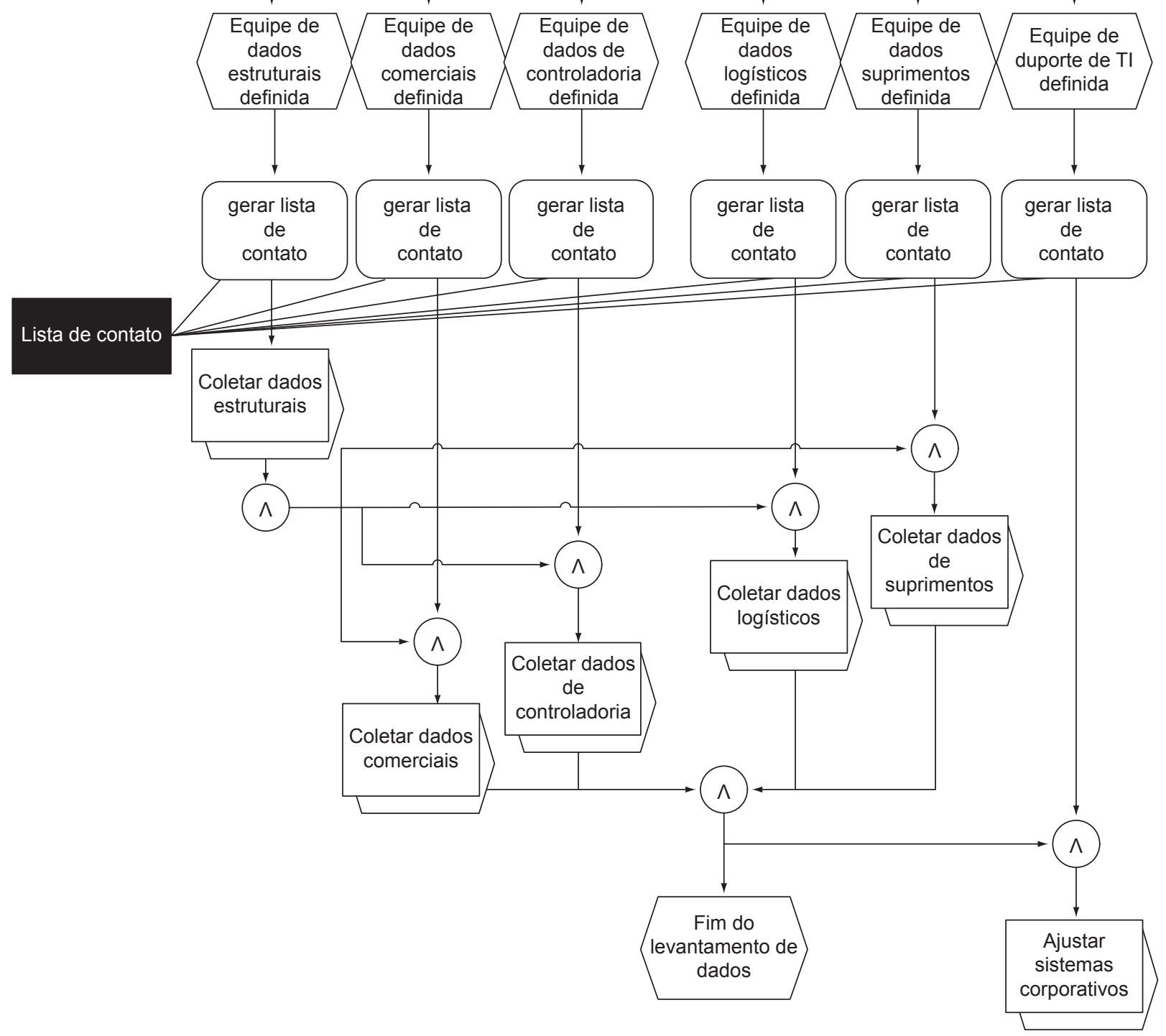

Figura 2. Exemplo de processo durante a fase de implantação do método: definição de equipes de trabalho. 


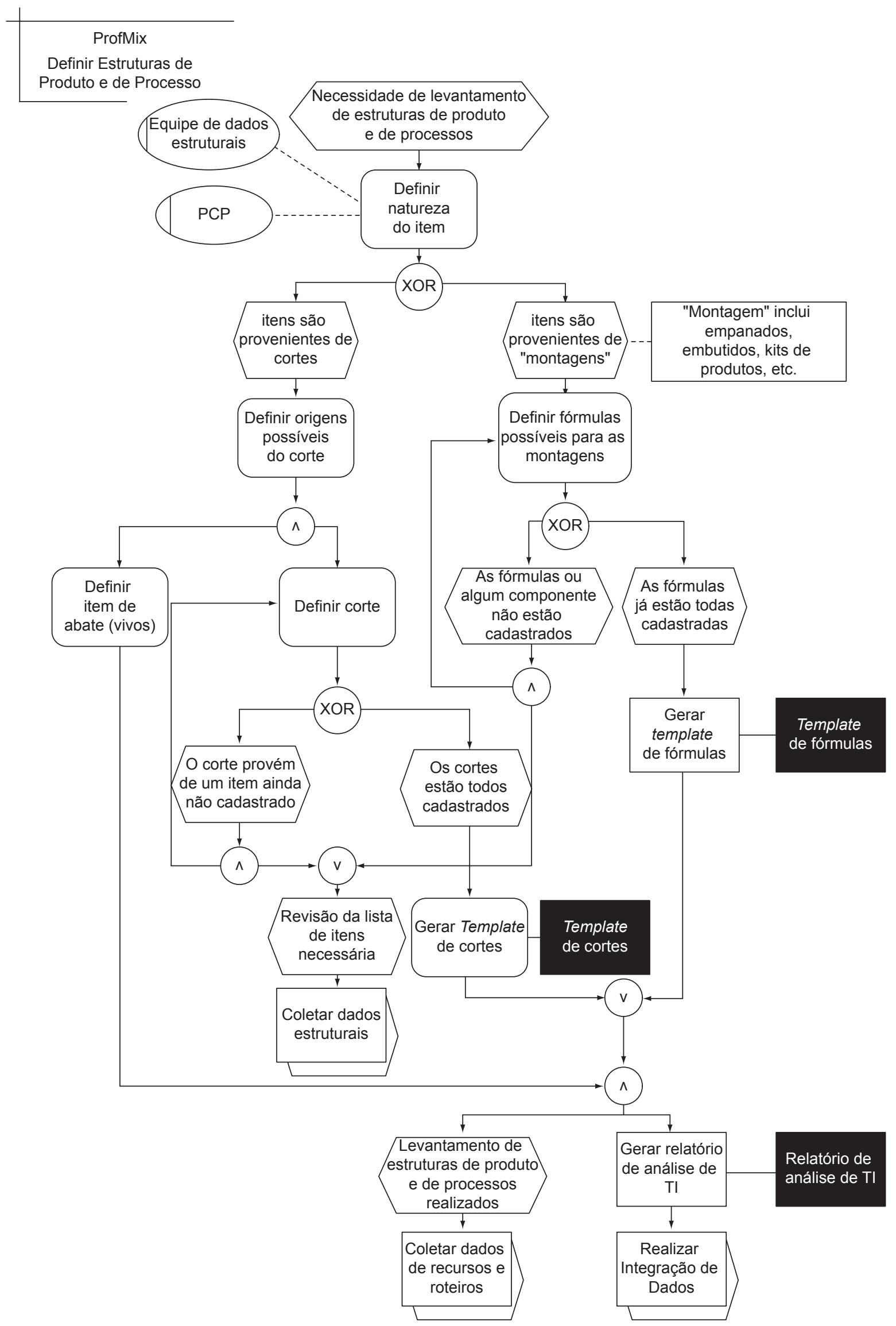

Figura 3. Exemplo de processo durante a fase de implantação do método: definição de estruturas de produtos. 
ferramenta de otimização de mix. Conforme mencionado anteriormente, para a manipulação da massa de dados, geração do modelo de otimização e conversão dos resultados ótimos na forma de um mix de produção foi utilizada a ferramenta ProfMix. Uma imagem dessa ferramenta é apresentada na Figura 4, evidenciando a estrutura de cortes com decisões compulsórias e alternativas.

O software selecionado incorpora as características de orientação ao mercado e flexibilidade de visão estratégica, suportado por conceitos da Teoria das Restrições, gerando um plano mestre otimizado de produção que respeita a orientação estratégica da empresa e as capacidades produtivas, minimizando, por consequiência, inventários e despesas operacionais. O ProfMix permite a geração de cenários que podem ser comparados, dando, aos gestores, meios de analisar mais amplamente os impactos de diferentes estratégias, tais como:

- Criação de novos produtos ou eliminação de produtos existentes;

- Aumento ou diminuição de capacidade agregada de recursos críticos;

- Utilização de políticas de produção em que o abate é "puxado" pela demanda; e

- Uso de fórmulas alternativas para embutidos, empanados, etc.

Esses cenários podem ser comparados não apenas na forma de volumes de produção, mas por meio de indicadores financeiros, relevantes para as diretorias das empresas.

A próxima etapa da implantação do método proposto de definição de mix ótimo de produção é o mapeamento adequado dos processos de gestão que interagem com a nova ferramenta. Essa visão se insere no método pro-

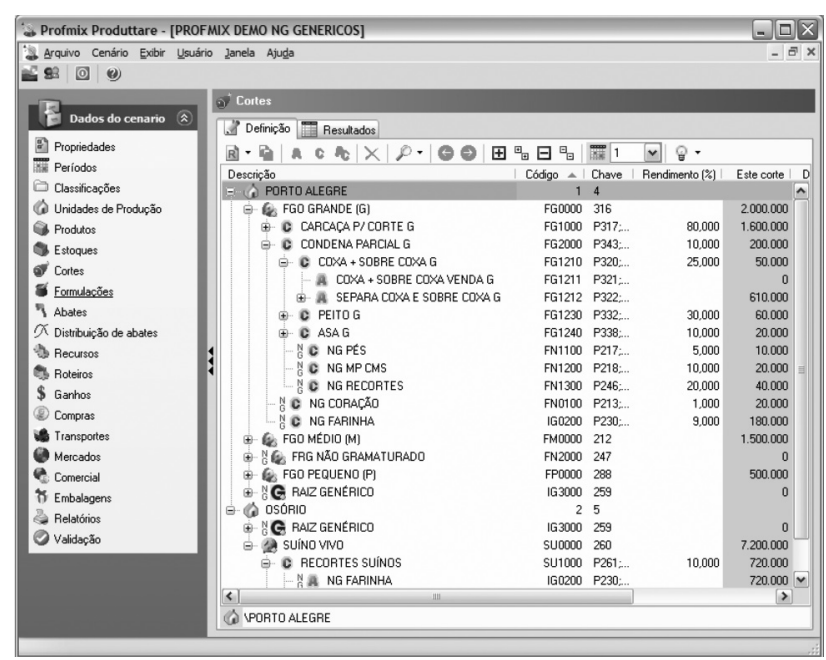

Figura 4. A ferramenta computacional ProfMix. posto no sentido de garantir que as dimensões da Gestão da Informação (pessoas, software, hardware, processos, comunicação) interajam adequadamente, mantendo o método de otimização de mix em pleno funcionamento. Como elemento de discussão deve-se considerar também a questão da centralização e da atualização dos dados apresentados à ferramenta de otimização de mix. Os dados necessários para o funcionamento de uma ferramenta desta natureza são originados em diferentes setores da empresa (diferentes unidades produtivas, diferentes departamentos, etc.). A manutenção destes dados exige a mobilização, o comprometimento e o apoio de diferentes profissionais da empresa, com claro entendimento da importância de seu papel no resultado projetado: dados que não correspondam à realidade da empresa potencialmente levarão a projeções irreais. Nesse sentido, uma etapa importante do método é a análise dos processos vigentes.

Os processos que devem ser analisados, tipicamente, são (Mansilha, 2005):

- Previsão e gestão de demanda;

- Criação de novo produto;

- Atualização de ganhos;

- Atualização de custos;

- Aquisição de novos equipamentos; e

- Geração do mix de produção.

Esses processos, depois de mapeados, devem ser redefinidos à luz da nova ferramenta de otimização de mix. O processo de "Simulação de cenários" não é comumente encontrado de maneira formalizada em indústrias do setor frigorífico, em função de ser extremamente laborioso. A presença de uma ferramenta com essa capacidade inserida no método proposto fomenta esse processo, viabilizando uma análise estratégica mais criteriosa e de forma mais flexível.

O redesenho dos processos completa o método de implantação proposto, permitindo a definição sistemática de um mix de produção orientado ao mercado e focado na maximização da receita líquida das empresas, com base nas efetivas capacidades produtivas. A implantação somente poderá ser considerada bem sucedida se o planejamento otimizado de produção tornar-se uma atividade cotidiana da empresa.

Do ponto de vista operacional, o uso de um particular aplicativo, responsável por dar o suporte computacional ao método de otimização de mix de produção, interfere na forma como o processo de implantação deverá ser executado. Dessa forma, o ProfMix foi desenvolvido de modo a permitir uma ampla visão dos conceitos anteriormente abordados, bem como diversos relatórios operacionais e gerenciais. A Figura 5 apresenta extratos da interface do ProfMix, em que se pode observar (a) 
as estruturas de itens formulados, (b) os recursos CCR e sua ocupação, (c) diferentes tipos de relatórios e (d) um exemplo de relatório de alinhamento às diretrizes comerciais.

Os benefícios do uso de uma abordagem hierárquica e otimizante da produção podem ser divididos em tangíveis e intangíveis. A aplicação prática da abordagem proposta e com o uso da ferramenta ProfMix em um frigorífico de suínos resultou, após 6 meses de uso, em (Baldissera, 2003):

- Incremento de $15 \%$ da margem de contribuição média em função do mix sugerido;

- Melhor aproveitamento das matérias-primas, com redução das necessidades de compra de carnes de terceiros em $50 \%$, considerados os mesmos níveis de atendimento; e

- Balanceamento de estoques, com redução de $20 \%$ do volume, em função do melhor aproveitamento dos cor-
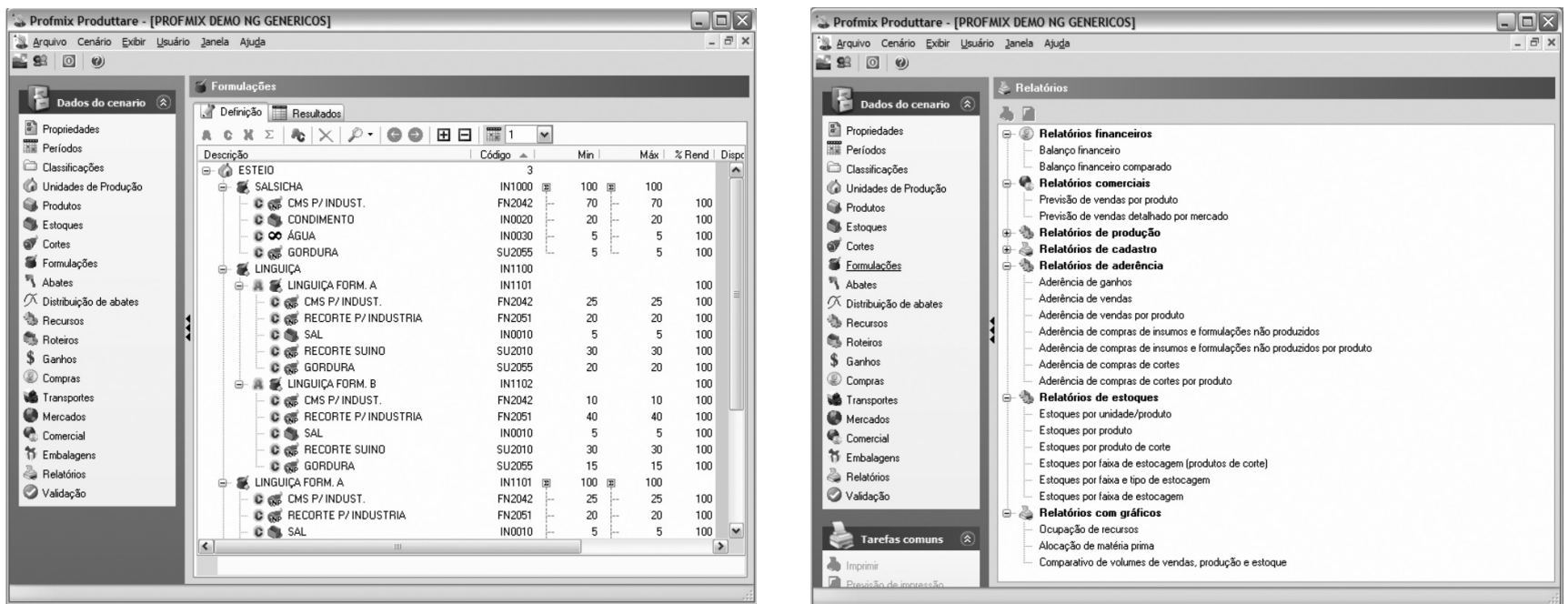

tes e da seleção de fórmulas adequadas às disponibilidades de carnes.

Do ponto de vista qualitativo, a aplicação da abordagem proposta em quatro empresas do setor permitiu observar:

- Qualificação do processo decisório, que passa a ser sistemático e analítico, aproximando as áreas comercial e de produção;

- Maior entendimento dos processos produtivos, com visão mais detalhada das inter-relações sistêmicas de suas subpartes, bem como dos impactos das alterações realizadas sobre essas partes. Por exemplo, a alteração da capacidade de um recurso considerado ccr pode ser avaliada do ponto de vista do impacto geral de desempenho quanto ao atendimento dos clientes ou do benefício econômico gerado, servindo como subsídio para a avaliação do investimento;
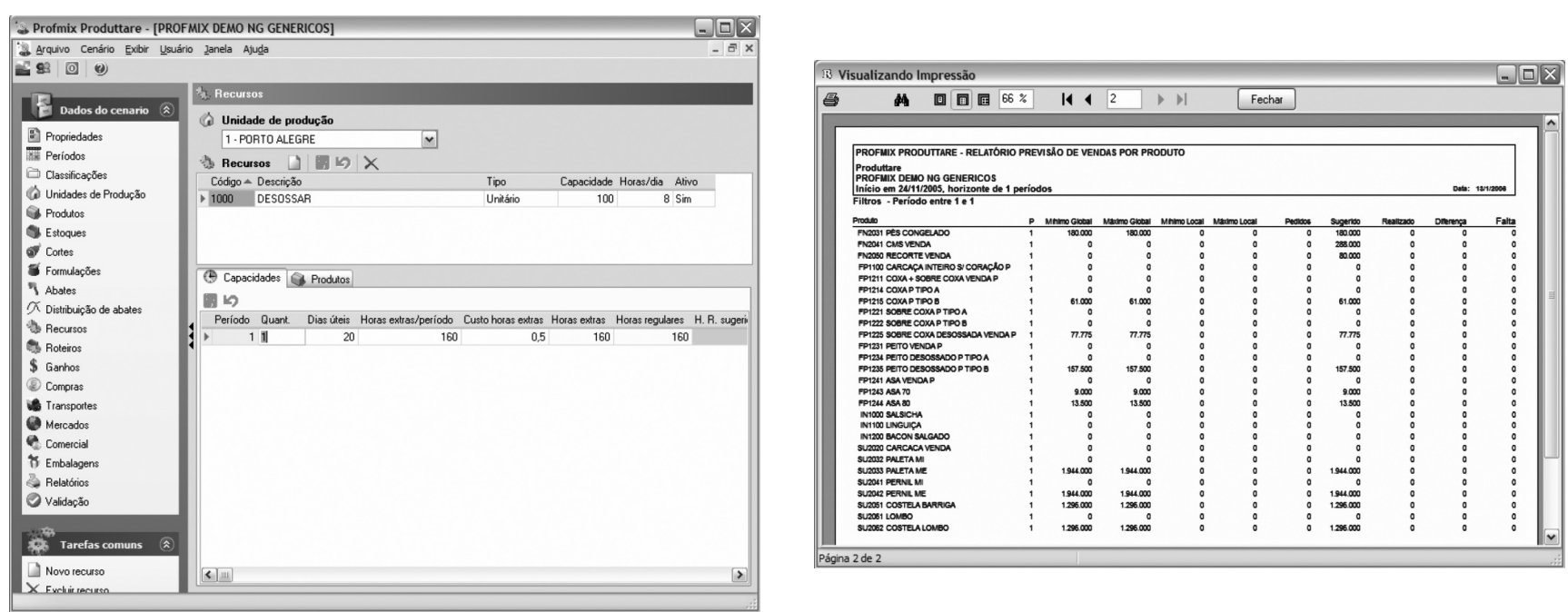

Figura 5. Extrato das interfaces e informações apresentadas pela ferramenta ProfMix. 
- Sistematização dos dados de produção, por seu cadastro e de sua manutenção e constante atualização, evitando redundâncias ou ambigüidades; e

- Aprendizagem da organização no que tange à gestão do processo produtivo e de vendas, pelo repasse de conhecimentos, da motivação dos profissionais da empresa envolvidos no projeto e da busca de benefícios coletivos.

No processo de implantação de uma abordagem ampla e sistêmica como a proposta neste artigo, certos fatores podem contribuir negativamente para a obtenção de resultados como os acima apresentados. Ao longo das quatro aplicações realizadas, os seguintes fatores foram identificados como críticos:

- Desconhecimento de conceitos de gestão da produção e de otimização por profissionais da empresa, uma vez que a abrangência da pesquisa operacional não é de domínio público em ambientes produtivos;

- Indisponibilidade de dados na forma adequada ou em forma estruturada; $\mathrm{e}$

- Necessidade de quebra de paradigmas, principalmente em relação à interpretação de resultados que beneficiam a empresa e não áreas ou unidades específicas, ou que alteram a sequiência histórica do modo de operação de determinadas áreas da empresa.

As aplicações realizadas permitiram identificar um conjunto de ações de suporte destinadas a minimizar os efeitos ou a ocorrência desses fatores críticos:

- Repasse de conhecimentos por meio de workshops e do acompanhamento da ação de profissionais-chave na geração de dados e na interpretação dos resultados gerados pela ferramenta;

- Desenvolvimento de esforços no desenvolvimento, na organização e na atualização sistemática de cadastros para itens de produção, recursos, tempos e produtividades;

- Acreditação dos resultados, pela busca constante e aprofundada de comportamentos diversos dos espera- dos, tais como produtividades excessivas ou demasiadamente baixas; e

- Acompanhamento de reuniões de gestão e avaliação detalhada das decisões tomadas, eventualmente com análise de cenários alternativos e avaliação do impacto de pressupostos no desempenho geral da organização.

\section{Conclusões}

O Brasil possui uma posição estratégica importante no setor frigorífico, a qual deve manter-se consolidada e, simultaneamente, permitir um aumento de sua participação no mercado mundial de carnes. Essa posição estratégica depende de inúmeros fatores, mas que certamente perpassam a orientação às exigências dos mercados consumidores e a racionalização do aproveitamento dos recursos e matérias-primas disponíveis.

A otimização de mix de produção, se bem utilizada, é uma estratégia que permite atender a esses objetivos. Para tanto, não se pode fundamentá-la somente no uso de um ou de um conjunto de ferramentas de software. Tampouco a otimização de mix de produção pode estar vinculada somente a um olhar definido sobre o ambiente interno das empresas.

O método proposto descreve elementos que devem ser considerados na implantação de uma estratégia de otimização de produção, observando que ela deve ser estrategicamente orientada ao mercado e ter indicadores adequados - fundamentados na Teoria das Restrições - para direcionar adequadamente os esforços de melhor uso dos recursos de produção. Além disso, o método contempla uma preocupação com a lógica de processos que se destinem não apenas às etapas iniciais de coletas de dados, mas à manutenção desses dados período após período, tornando efetiva sua realização. Uma última parte necessária, mas não suficiente, é o uso de um software capaz de produzir um mix otimizante que seja alinhado com essa orientação estratégica ao mercado.

\section{Referências Bibliográficas}

ABEF. Abef Online. Estatísticas de 2004. Disponível em <http://www.abef.com.br>. Acesso em: 22 de abril, 2005.

ABEF. Abef Online. Estatísticas de 2005 e 2006. Disponível em <http://www.abef.com.br>. Acesso em: 21 de dezembro, 2005.

ABIPECS. Relatório Anual 2004. Disponível em <http:// www.abipecs.com.br>. Acesso em: 31 de março, 2005.

ANTUNES JR, J. A. V.; RODRIGUES, L. H.; PIZZATO, F. A construção do plano agregado estratégico de produção
- uma abordagem crítica e operacional. In: CONGRESSO BRASILEIRO DE CUSTOS, 8, São Leopoldo, 2001. Anais...CD-ROM.

BALDISSERA, A. L. Um Estudo da Aplicação da Pesquisa Operacional para Otimizar Resultados em uma Agroindústria: o caso da unidade de negócios de suínos da Chapecó Companhia Industrial de Alimentos. Dissertação de Mestrado - Universidade Federal de Santa Catarina Florianópolis: UFSC, 2003.

BAYOU, M. E.; REINSTEIN, A. Analyzing the ProductMix Decision by Using a Fuzzy Hierarchical Model. 
Managerial Finance. Patrington, v. 31, n. 3, 2005, p. 3549. 2005 .

BERCHT, M. Plano Agregado Estratégico de Produção. Porto Alegre: UFRGS - Universidade Federal do Rio Grande do Sul, 1996. Dissertação de Mestrado.

CHASE, R. B.; AQUILANO, N. J. Production and Operation Management: Manufacturing and Services, $7^{\mathrm{a}} \mathrm{ed}$ Chicago: Irwin, 1995.

CORBETT, T. Throughput Accounting. Great Barrington, MA: North River Press, 1998.

DAVENPORT, T. H. Reengenharia de Processos. Rio de Janeiro: Campus, 1994.

GOLDRATT, E. M.; Cox, J. A Meta. São Paulo: Educator, 1986.

GOLDRATT, E. M. Mais que Sorte... Um Processo de Raciocínio. São Paulo: Educator, 1994.

LEA, B. R.; FREDENDALL, L. D. The Impact of Managemente Accounting, Product Structure, Product Mix Algorithm, and Planning Horizon on Manufacturing Performance. International Journal of Production Economics, Elsevier, v. 3, n. 79, p. 279-299, 11 October 2002.
LOW, J. Do We Really Need Product Costs? The Theory of Constraints Alternative. Corporate Controller, v. 1, n. 5, p. 26-36, 1992.

MANSILHA, R. B. Desenvolvimento de uma abordagem de processos para operacionalização do Plano Mestre Otimizado de Produção para indústria frigorífica. Dissertação de Mestrado - Universidade do Vale do Rio dos Sinos, São Leopoldo: UNISINOS, 2005.

OENNING, V. Decisões sobre mix de produção em indústrias de produção conjunta: uma abordagem a partir da Teoria das Restrições e da Pesquisa Operacional. São Leopoldo: UNISINOS - Universidade do Vale do Rio dos Sinos, 2004. Dissertação de Mestrado.

RODRIGUES, L. H. Developing an Approach to Help Companies Synchronise Manufacturing. Tese de Doutorado - Lancaster University, Inglaterra, 1994.

RODRIGUES, L. H.; CASSEL, R. A.; VACCARO, G. L. R.; PELLEGRIN, I.; BECKER, F. Desenvolvimento de um Aplicativo Computacional para Seleção de Mix de Produtos em Empresas com Processos Produtivos Divergentes. In: CLADEA - CONGRESSO LATINO-AMERICANO DAS ESCOLAS DE ADMINISTRAÇÃO 37. Porto Alegre, 2002. Anais...CD-ROM.

\section{STUDY OF THE IMPLEMENTATION OF A PRODUCTION MIX OPTIMIZER FOR THE BEEF SECTOR}

\section{Abstract}

This paper proposes the implementation of a mix optimization tool for the beef sector, particularly for meat packing plants. The implementation of a mix optimization tool involves different areas of the organization, since this is a systemic tool for creating a market-oriented master production plan based on aggregate finite capacities, which optimizes the company's profitability. The article discusses the dimensions involved in the implementation of such a project and the main benefits resulting from it.

Keywords: mix optimization, production planning, divergent processes. 
